\title{
Prescriptivo y Simulación. Etiquetaje de la eficiencia energética del edifício comercial "Torre Plaza Shopping", Passo Fundo, RS, Brasil en función de las dos metodologías de la reglamentación brasileña
}

Prescriptive and Simulation Methodologies. Labeling of the energetic efficiency of a commercial building, "Tower Plaza Shopping", Passo Fundo, RS, Brazil, in accordance with the mentioned methods under the respective Brazilian regulation - RTQ - C

Letiane Benincá

Mestre em Arquitetura, Universitat Politècnica de Catalunya Rua 20 de Setembro, 377 apto 501, Centro, Passo Fundo, Rio Grande do Sul, Brasil Ifbeninca@hotmail.com

Eduardo Grala da Cunha Pós-Doutor em Arquitetura e Urbanismo, professor na Universidade Federal de Pelotas Rua Benjamin Constant, 1359, Pelotas, Rio Grande do Sul, Brasil eduardo.grala@ufpel.edu.br

Alexis Aguilar Sanchez Doutor em Arquitetura, professor do Máster em Arquitetura na Universitat Politècnica de Catalunya, Avinguda Diagonal, 649, Barcelona, Espanha. alexis.aguilar@upc.edu 
Resumen

El trabajo estudia la panorámica de la Eficiencia Energética, tomando em cuenta - y enfatizando - el programa brasileño del INMETRO, PROCEL Edifica, contenido en la norma Requisitos Técnicos de Calidad para el Nivel de Eficiencia Energética de Edificios Comerciales, de Servicios y Públicos (RTQ C). Con esse tema en mente, han sido consultadas algunas normativas mundiales y ha sido hecho un profundo estudio de la reglamentación brasileña vinculada.

Para la mejor comprensión de la normativa, ha sido elegida una edificación comercial emblemática, en altura, en la ciudad de Passo Fundo, RS, Brasil, para ilustrar la perspectiva del programa. El edificio ha sido clasificado de acuerdo a lãs dos metodologías presentadas por la reglamentación respectiva: el Método Prescriptivo y el Método de Simulación. Se ha podido así investigar las peculiaridades de cada metodología y de la propia reglamentación en sí, basándose en el caso de estudio especifico: edificios comerciales, en altura, en el sur de Brasil, resultando en la clasificación del nivel de eficiencia energética de la edificación de referencia.

Finalmente, después de haberse hecho todos los levantamientos, cálculos y simulaciones, bajo la luz de la clasificación de la edificación por los dos métodos, se han podido comparar los resultados. Se llega así a la conclusión de que el Método Prescriptivo es menos preciso y necesita de gran dedicación por parte del evaluador para elaborar todos los cálculos y levantamientos necesarios. Sin embargo, es uma herramienta más rápida y accesible a los profesionales. La Simulación, por otro lado, es una metodología más precisa y llega a niveles de análisis que el Método Prescriptivo no alcanza; no obstante, es todavía una herramienta poco conocida em Brasil y que necesita de un gran aporte financiero inicial, lo que la mantiene limitada por tanto, a grandes estudios de Construcción Civil o a Laboratorios Universitarios.

Palabras clave: Eficiencia Energética, Etiquetaje, Reglamentación brasileña. 
Abstract

The work studies the panoramic of the Energetic Efficiency, emphasizing the Brazilian program of the INMETRO, PROCEL "Edifica", contained in the norm Technical Requirements of the Quality for the Level of Energetic Efficiency of Commercial, Service, and Public Buildings (RTQ - C). With this subject in mind, it was made a worldwide research consulting some rules related to, and after that, it was made a profound study of the Brazilian correlate regulation.

For the best comprehension of the focused rule, it was chosen a commercial edifice, in height, in the city of Passo Fundo, RS, Brazil, to illustrate the perspective of the program. The building was classified under the application of the two methodologies presented by the Brazilian regulation: the Prescriptive and the Simulation Methods. It could be then made an analysis of the peculiarities of each methodology and the Brazilian regulation in itself, based on the specific case of study: a commercial building, in height, in the South of Brazil, ensuing in the classification of the level of energetic efficiency of the mentioned edifice.

Finally, after all data accomplishment, calculations and simulations have been carried out, and the classification of the construction has finished using both methodologies in focus, the results could be then being compared. It was concluded that the Prescriptive Method is less precise than the other one and it needs more dedication to make all the calculations and necessary accomplishments; yet, it is a faster tool, very accessible for professionals. The Simulation Method, on the other hand, is more accurate, in awarding levels of analysis that the Prescriptive Method does not reach. It is a tool not so known in Brazil as well, needing a bigger initial investment that keeps it limited to top Construction Firms or to University Laboratories.

Keywords: Energy Efficiency, Labeling, Brazilian regulation 


\section{EL PROGRAMA BRASILEÑO DE ETIQUETAJE (PBE)}

Tiene como objetivos principales, proporcionar información que pueda ayudar a la toma de decisión en la compra de un equipamiento, que sea más eficiente que otro, así como el fomento de la competitividad industrial a través del proceso de inducción de la mejora continua, promovidos por la elección consciente de los consumidores.

La Etiqueta Nacional de Conservación de Energía (ENCE) es el principal producto del PBE ejecutado por el INMETRO. Su adhesión es voluntaria y realizada por sus representantes comerciales. La "ENCE" muestra la clasificación de la eficiencia energética de los productos, informando al consumidor, marca, modelo, energía consumida y las especificaciones técnicas del aparato a ser comprado.

\section{PROCEL EDIFICA - PROGRAMA NACIONAL DE EFICIENCIA ENERGÉTICA EN EDIFICACIONES}

Es un programa del INMETRO que tiene como plan de acción reglamentar la eficiencia energética en edificaciones. Inicialmente voluntario, visa construir las bases necesarias para racionalizar el consumo de energía en el Brasil. La idea del programa es promover el etiquetaje de edificios según la forma con que éste utiliza la energía eléctrica. Actúa de forma conjunta con el Ministerio de Minas y Energía, el Ministerio de las Ciudades, universidades, centros de pesquisa y entidades de las áreas gubernamental, tecnológica, económica y de desarrollo, además del sector de la construcción civil.

El programa, con base en la norma Requisitos Técnicos de la Calidad para el Nivel de Eficiencia Energética de edificios comerciales, de servicios y públicos (RTQ $-C)$, tiene como objetivo crear condiciones para etiquetar voluntariamente el nivel de eficiencia energética de edificios comerciales, de servicio y públicos, Tiene como su descripción general la de especificar métodos para la clasificación del nivel de eficiencia energética de edificaciones comerciales.

Puede aplicarse a edificios con un área total útil, mínima, de $500 \mathrm{~m}^{2}$ o con tensión de abastecimiento eléctrico igual o superior a 2,3 $\mathrm{kW}$, para edificios con acondicionamiento de aire, parcialmente y/o naturalmente ventilados. Es requisito para el análisis, que los edificios comerciales, que no sean acondicionados, deban comprobar por simulación que los ambientes de permanencia humana prolongada proporcionen temperaturas, dentro de la zona de conforto, durante ochenta por ciento (80\%) de las horas de uso al año.

Profundizando los conocimientos acerca del programa, la reglamentación presenta dos metodologías para la clasificación del nivel energético de las edificaciones. Los métodos conllevan gran trabajo con el atendimiento a los prerrequisitos generales y específicos de cada sección de la reglamentación y son explicados de forma esquemática a seguir:

Método Prescriptivo: procedimiento analítico donde son aplicadas ecuaciones que reciben como entrada informaciones relativas a las características de la envolvente de la estructura externa (arquitectónicas y constructivas), iluminación y acondicionamiento de aire. La puntuación obtenida determina la clasificación de la eficiencia energética del edificio evaluado, resumiéndose esta metodología, cómo muestra la figura 1.

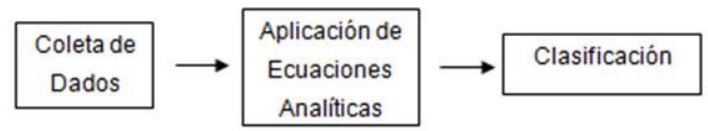

Fig. 1 - Esquema Método Prescriptivo

El método especifica niveles de eficiencia energética clasificados de $A$ (más eficiente) a $E$ (menos eficiente) y posee una división de pesos, conforme el ítem evaluado, que son:

Envolvente (Env) - peso de 30\%;

Sistema de lluminación (DPI) - peso de $30 \%$ y 
Sistema de acondicionamiento de Aire $(C A)$ - peso de $40 \%$.

La clasificación del edificio es calculada de acuerdo con la distribución de los pesos a través de la ecuación 1:

$$
\mathrm{PT}=0,30 \cdot\left\{\left(\text { EqNumEnv } \frac{\mathrm{AC}}{\mathrm{AU}}\right)+\left(\frac{\mathrm{APT}}{\mathrm{AU}} \cdot 5+\frac{\mathrm{ANC}}{\mathrm{AU}} \cdot \text { EqNumV }\right)\right\}+0,30 \cdot(\text { EqNumDPI })+0,40 \cdot\left\{\left(\text { EqNumCA. } \frac{\mathrm{AC}}{\mathrm{AU}}\right)+\left(\frac{\mathrm{APT}}{\mathrm{AU}} \cdot 5+\frac{\mathrm{ANC}}{\mathrm{AU}} \cdot \mathrm{EqNum}^{\mathrm{N} V}\right)\right\}+b_{0}^{1}
$$

(Ecuación 1)

Donde:

EqNumEnv: equivalente numérico de la envolvente/ estructura externa;

EqNumDPI: equivalente numérico del sistema de iluminación, identificado por la sigla DPI, de Densidad de Potencia de lluminación;

EqNumCA: equivalente numérico del sistema de acondicionamiento de aire;

EqNumV: equivalente numérico de ambientes no acondicionados y/o ventilados naturalmente (el cual tiene que ser verificado por la simulación; si ello no ocurre su valor es igual a 1);

APT: área útil de los ambientes de permanencia transitoria, desde que no acondicionados (pasillos, escaleras y baños);

ANC: área útil de los ambientes no acondicionados de permanencia prolongada, con comprobación de porcentual de horas ocupadas en conforto (POC) por ventilación natural a través de simulación;

AC: área útil de los ambientes acondicionados;

$\mathrm{AU}$ : área útil;

b: puntuación obtenida por las bonificaciones, que varía de 0 a 1.

Método de Simulación: consiste en comparar el desempeño término energético de la edificación real con las de referencia $(A, B, C$ y $D)$. Para tanto, es necesario realizar la simulación de los modelos (real y de referencia) por medio de un software especializado (DesignBuilder versión 2.0.4.001), esquematizándose la metodología, en la figura 2.

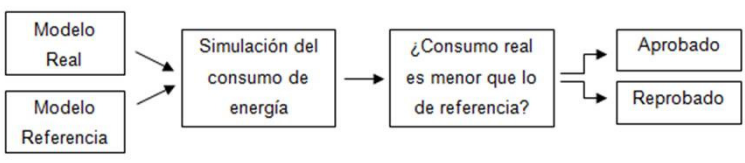

Fig. 2 - Esquema Método Simulación

La puntuación total (PT) de edificios totalmente simulados será calculada por la ecuación:

$$
\mathrm{PT}=\mathrm{EqNums}+b_{0}^{1}
$$

\section{(Ecuación 2)}

Donde:

EqNums: equivalente numérico obtenido a través de la simulación;

b: puntuación obtenida por las bonificaciones, que varía de 0 a 1.

Bonificaciones: son medidas que priorizan la utilización de estrategias que disminuyan el impacto ambiental. Alcanzan, como máximo, un (1) punto sumado a la puntuación general; este punto es atribuido a iniciativas justificadas y con economía comprobada, que pueden ser: el uso racional del agua, con economía mínima de veinte por ciento (20\%); uso de calefacción de agua por placas solares, generando economía mínima de sesenta por ciento (60\%); utilización de fuentes renovables de energía, la que representa una economía mínima de diez por ciento (10\%); uso de la cogeneración (reutilización de calor en procesos termodinámicos, puede ocurrir en forma de vapor, agua caliente y/o frío, por ejemplo: turbinas de vapor, de gas y motores de 
combustión interna), generando una economía mínima de treinta por ciento (30\%) e innovaciones que promuevan la eficiencia energética, con una economía mínima del treinta por ciento (30\%).

A partir de la puntuación general, generada por la ecuación, se puede clasificar el edificio en:

\begin{tabular}{c|c}
\hline Puntuación & Clasificación \\
\hline$\geq 4,5$ a 5 & $\mathrm{~A}$ \\
\hline$\geq 3,5 \mathrm{a}<4,5$ & $\mathrm{~B}$ \\
\hline$\geq 2,5 \mathrm{a}<3,5$ & $\mathrm{C}$ \\
\hline$\geq 1,5 \mathrm{a}<2,5$ & $\mathrm{D}$ \\
\hline$<1,5$ & $\mathrm{E}$ \\
\hline
\end{tabular}

Tabla 1 - Puntuación y Clasificación equivalente

La reglamentación posibilita la clasificación del edificio completa o parcialmente, conforme sea necesario.

\section{METODOLOGÍA DE ANÁLISIS}

Para llegar a los resultados adquiridos fueron tomadas en consideración las bases de las formas de arquitectura pasiva y activa, de donde salieron los análisis vinculados a la edificación elegida, que son:

Análisis de los elementos de la envolvente del edificio, paredes, cubiertas, desde la transmisión térmica, absorción solar, inercia térmica $y$ aislamiento, de los datos de iluminación existentes y de la parte eléctrica construida y por fin, datos de los aparatos activos existentes (acondicionamiento de aire, estufas, lámparas, etc).

Fueran hechas diversas visitas al edificio, evidenciándose todos los condicionantes pertinentes a la construcción, colectándose los datos de equipamientos, materiales existentes, sistemas de iluminación y acondicionamiento de aire, así como sistemas pasivos y la utilización de elementos arquitectónicos relevantes para el estudio.

Posteriormente, ha sido hecho un análisis general del clima y de las condiciones preexistentes; ambientales, del lugar. Después de haber colectado todos los datos, fueron aplicadas las dos metodologías de cálculo para la clasificación del nivel de eficiencia energética de la edificación seleccionada, presentadas en el RTQ - C: el Método Prescriptivo y el Método de Simulación. Habiéndose clasificado parcialmente el edificio, se evalúa la "Torre Plaza Shopping", haciéndose, sin embargo, hincapié que la envolvente de la estructura externa del edificio (Shopping y Torre) fue evaluada como un todo, por ésta ser una estructura única, debiendo así ser estudiada integralmente. Los demás parámetros del sistema de iluminación y de acondicionamiento de aire, fueron estudiados aisladamente, apenas para la parte del edificio utilizada como escritorios, pudiendo así, verificarse los resultados específicos de cada etapa descrita en la norma y los aportes y limitaciones de cada método.

\section{CONDICIONANTES CLIMÁTICOS Y UBICACIÓN DE LA EDIFICACIÓN}

El objeto de estudio está localizado en la ciudad de Passo Fundo, que pertenece al Estado de Rio Grande del Sur, Brasil. Éste limita con la Repúblicas Argentina al oeste, con la República Oriental del Uruguay al sur y con el Estado de Santa Catarina al norte, teniendo su costa bañada por el Océano Atlántico al este. Situado en las coordenadas de longitud y latitud de $28^{\circ} 15^{\prime} 46^{\prime \prime} \mathrm{S}$ y $52^{\circ} 24^{\prime} 25^{\prime \prime}$, la ciudad de referencia se encuentra a una altitud de $680 \mathrm{~m}$ respecto al nivel del mar.

Según la clasificación climática de Köppen, la región posee un clima meso-termal, húmedo, templado, del tipo subtropical húmedo ( $\mathrm{Cfa}$ ), caracterizado por temperaturas medias que en los meses más fríos, varían entre $18 \circ \mathrm{C}$ y $0 \circ \mathrm{C}$ y en el mes más caluroso pueden llegar o ultrapasar los 30 ㅇ. .

En razón de la posición geográfica del Estado, éste sufre la influencia de los vientos que vienen tanto de la zona tropical cuanto de la zona polar austral; el calor del verano es causado por los vientos cálidos procedentes del norte. Como sucede en otras regiones, el clima presenta variaciones locales 
causadas por la influencia de factores tales como la altitud y la distancia del océano Atlántico.

Posee una temperatura media anual de $17,5 \mathrm{C}$, teniendo como mes más caluroso el de enero y el mes más frio el de junio; la región se caracteriza por tener veranos moderadamente calurosos e inviernos húmedos y amenos; en los meses más fríos es común la formación de heladas y ocasionalmente la ocurrencia de nieve.

Tiene las lluvias bien distribuidas al largo del año, siendo el mes de septiembre el de mayor volumen (206.8 mm) y abril el de menor (118.2 mm). La humedad relativa del aire se mantiene entre $67 \%$ en los meses de noviembre y diciembre y $76 \%$ en el mes de junio.

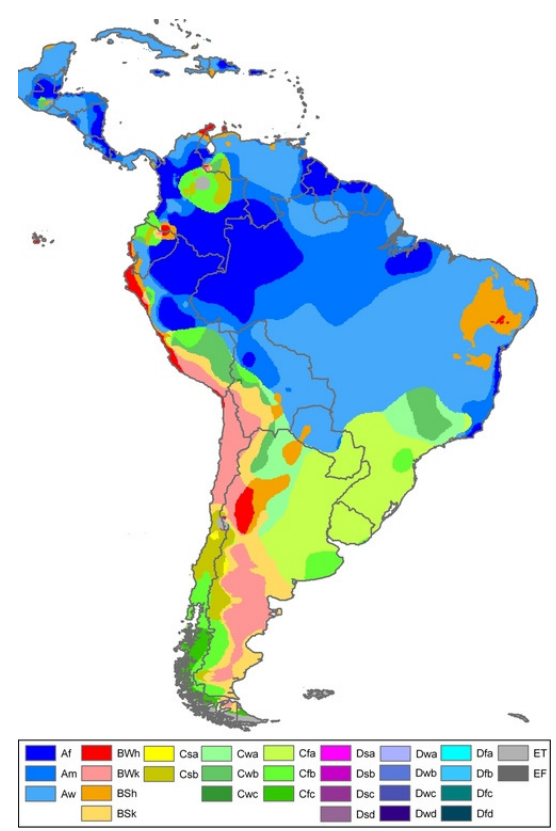

Los vientos predominantes vienen de la dirección $\mathrm{NE}$, con velocidades medias de $3,80 \mathrm{~m} / \mathrm{s}$ y $4,70 \mathrm{~m} / \mathrm{s}$, con vientos secundarios provenientes del SE. La velocidad de los vientos en el período de invierno genera una sensación no confortable, pero específicamente cuando éstos provienen del E; ya en el periodo estival favorecen la ventilación y el consiguiente conforto, por tener una velocidad superior a $2 \mathrm{~m} / \mathrm{s}$.

Para comprender de forma general los datos climáticos del lugar, éstos fueron inseridos en el Diagrama Bioclimático de Givoni.

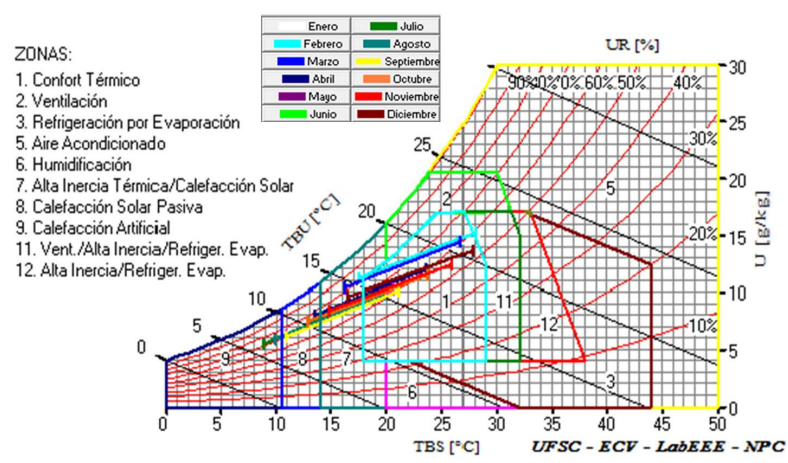

Fig. 3 - Diagrama bioclimático de Givoni Software Analisys Bio (UFSC 2011)

Analizado el Ábaco de Givoni, se pueden destacar algunas estrategias para mejorar el conforto térmico del usuario en la zona elegida. Para todos los meses del año la masa térmica minimiza la falta de éste; en el mes más caluroso, enero, la utilización de la ventilación de conforto; para los meses más fríos, de junio a agosto, la utilización de calefacción artificial.

El objeto de estudio elegido, fue el edificio comercial "TORRE PLAZA SHOPPING", en la ciudad de Passo Fundo, Rio Grande del Sur, Brasil. Está ubicado en la calle Paissandú, sito en el número 1515 , esquina con las calles XV de Noviembre y la Avenida 7 de Setiembre, en el centro de la ciudad.

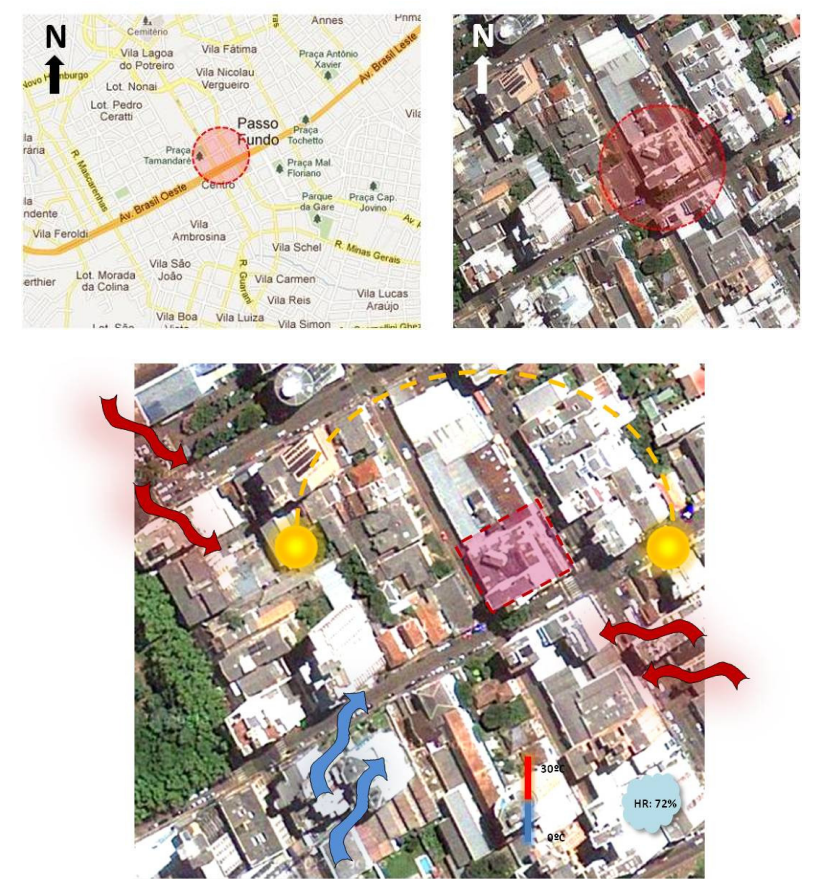

Fig. 4 - Ubicación del edificio en la ciudad Fuente: Google maps (2011)

Por lo expuesto, el esquema "ut infra" refuerza la comprensión de los condicionantes climáticos del lugar, ayudando a tener una mejor 
visibilidad de las posibles estrategias arquitectónicas que prioricen el conforto del usuario.

\section{APLICACIÓN DE LAS METODOLOGÍAS EN EL EDIFICIO COMERCIAL "TORRE PLAZA SHOPPING"}

La reglamentación enumera prerrequisitos generales, que deben ser atendidos en función de toda la edificación; éstos son: los circuitos eléctricos, los sistemas de calefacción de agua y los ascensores. Para el caso de estudio tenemos:

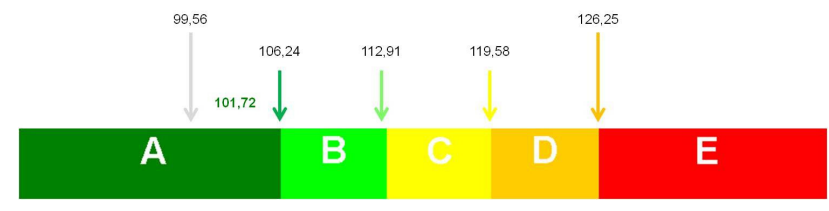

Fig. 5 - Escala del ICenv del edificio

Fueron verificados los prerrequisitos específicos para la estructura externa, que son:

Tabla 3 - Resultados de los prerrequisitos específicos de la envolvente/estructura externa

\begin{tabular}{|c|c|c|c|}
\hline $\begin{array}{c}\text { Prerrequisitos } \\
\text { específicos }\end{array}$ & Valor & EqNum & Clasificación \\
\hline \multirow[t]{2}{*}{$\begin{array}{l}\text { Transmisión } \\
\text { térmica }\end{array}$} & $\begin{array}{c}\text { Upar: } \\
2,49 \mathrm{~W} / \\
\left(\mathrm{m}^{2} \cdot \mathrm{K}\right)\end{array}$ & 3 & $\mathrm{C}$ \\
\hline & $\begin{array}{c}\text { Ucob: } \\
2,41 \mathrm{~W} / \\
\left(\mathrm{m}^{2} \cdot \mathrm{K}\right)\end{array}$ & 1 & $E$ \\
\hline \multirow{2}{*}{$\begin{array}{c}\text { Colores y } \\
\text { absorción de las } \\
\text { superficies }\end{array}$} & $\alpha=0,34$ & 5 & $A$ \\
\hline & $\alpha=0,85$ & 1 & $E$ \\
\hline $\begin{array}{c}\text { Iluminación } \\
\text { cenital }\end{array}$ & $\begin{array}{c}\mathrm{PAZ}<5 \% \\
\text { (verificar } \\
\text { por } \\
\text { simulació } \\
\mathrm{n} \text { ) }\end{array}$ & 3 & $\mathrm{C}$ \\
\hline Clasificación & & 1 & $E$ \\
\hline \multicolumn{4}{|c|}{$\begin{array}{l}\text { Resulta así en la reclasificación de la } \\
\text { envolvente/estructura externa del edificio en un } \\
\text { nivel } E \text { (EqNum }=1 \text { ), porque la transmisión térmica } \\
\text { de la cubierta del edificio de despachos es muy alta } \\
\text { (mismo que sea pequeña), lo que muda la } \\
\text { clasificación de la edificación, que por los cálculos } \\
\text { sería de } A \text {. }\end{array}$} \\
\hline
\end{tabular}

Iluminación: comparando la potencia instalada de la edificación, de $7.502 \mathrm{~W}$, con las potencias limites, conforme la tabla a seguir indicada, se verifica que la clasificación del sistema de iluminación es $A$; pero se deben evaluar los prerrequisitos específicos.

Tabla 4 - Límite máximo aceptable de densidad de potencia de iluminación (DPIL) para el nivel de eficiencia pretendido - Método del área del edificio

\begin{tabular}{|c|c|c|c|c|}
\hline F unçäo do Edifíicio & 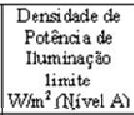 & $\begin{array}{c}\text { Densidade de } \\
\text { Potência de } \\
\text { Iluminação } \\
\text { linite } \\
\text { W/m } \mathrm{m}^{2} \cong \text { Nivel B) }\end{array}$ & \begin{tabular}{|c|} 
Densidade de \\
Potencia de \\
iluninaça \\
linite \\
$\mathrm{W}^{2} \mathrm{~m}^{2} \mathrm{~N}$ Nive $\mathrm{Cl}$ \\
\end{tabular} & $\begin{array}{c}\text { Densidade de } \\
\text { Potencia de } \\
\text { Iluminaf̧a } \\
\text { limite } \\
\text { W/m } \mathrm{m}^{2} \text { Nivel D) }\end{array}$ \\
\hline Escritório & 9,7 & 11,2 & 12,6 & 14,1 \\
\hline
\end{tabular}

Fuente: INMETRO (2011)

Multiplicando el total del área iluminada del edificio (999, $96 \mathrm{~m}^{2}$ ) por la DPI de cada nivel, se encuentran las potencias-limite, conforme la tabla:

Tabla 5 - Cálculos DPIL

\begin{tabular}{c|c|c|c|c}
\hline Función & $\begin{array}{c}\text { DPI } \\
\text { Nivel A }\end{array}$ & $\begin{array}{c}\text { DPI } \\
\text { Nivel B }\end{array}$ & $\begin{array}{c}\text { DPI }_{\mathrm{L}} \\
\text { Nivel C }\end{array}$ & $\begin{array}{c}\text { DPI } \\
\text { Nivel D }\end{array}$ \\
\hline Despach & 9,7 & 11,2 & 12,6 & 14,1 \\
os & $\mathrm{W} / \mathrm{m}^{2}$ & $\mathrm{~W} / \mathrm{m}^{2}$ & $\mathrm{~W} / \mathrm{m}^{2}$ & $\mathrm{w} / \mathrm{m}^{2}$ \\
\hline Totales & $\mathbf{9 . 6 9 9 , 6}$ & $\mathbf{1 1 . 1 9 9}$ & $\mathbf{1 2 . 5 9 9}$, & $\mathbf{1 4 . 0 9 9}$ \\
& $\mathbf{0}$ & $\mathbf{5 5}$ & $\mathbf{5 0}$ & $\mathbf{4 3}$ \\
& $\mathbf{W} / \mathrm{m}^{\mathbf{2}}$ & $\mathbf{W} / \mathrm{m}^{\mathbf{2}}$ & $\mathbf{W} / \mathrm{m}^{\mathbf{2}}$ & $\mathbf{W} / \mathrm{m}^{\mathbf{2}}$ \\
\hline
\end{tabular}

Verificando los prerrequisitos para el sistema de iluminación, tendremos:

Tabla 6 - Prerrequisitos específicos para iluminación

\begin{tabular}{c|c|c}
\hline \multicolumn{2}{c|}{ PRES REQUISITOS ESPECIFICOS } & Clasificación \\
\hline $\begin{array}{c}\text { División de } \\
\text { circuitos }\end{array}$ & Posee & $\mathrm{A}$ \\
\hline $\begin{array}{c}\text { Contribución } \\
\text { de la luz } \\
\text { natural }\end{array}$ & No Posee & $\mathrm{C}$ \\
\hline $\begin{array}{c}\text { Apagamiento } \\
\text { automático } \\
\text { del sistema de } \\
\text { iluminación }\end{array}$ & $\begin{array}{c}\text { No posee } \\
\text { No tiene } \\
\text { ambientes } \\
\text { mayores de } 250 \\
\text { m2. }\end{array}$ & - \\
\hline \multicolumn{2}{c|}{ Clasificación } & C \\
\hline
\end{tabular}

Por la potencia instalada la edificación, ésta es clasificada como de nivel $A$, pero al no cumplir con los prerrequisitos específicos, alcanza una clasificación $C$, con EqNum = 3 .

Aire acondicionado: los acondicionadores de aire son todos del tipo Split con eficiencia evaluada por el 
PBE/INMETRO y de acuerdo con las normas brasileñas y/o internacionales. Después de verificar todos los equipamientos utilizados en el edificio, encontramos el EqNumAC = 3,57, equivalente $a$ clasificación $B$. Verificando el prerrequisito específico para ese ítem, se observa que los equipos no la atienden, ya que la parte externa (condensador) de los Splits no poseen sombra permanente.

\section{CLASIFICACIÓN GENERAL POR EL MÉTODO PRESCRIPTIVO:}

Para la clasificación de la eficiencia de la edificación, se debe inserir todas las variables encontradas en la ecuación de la Puntuación Total.

$$
\begin{aligned}
& \mathrm{PT}=0,30 \cdot\left\{\left(\text { EqNumEnv. } \frac{\mathrm{AC}}{\mathrm{AU}}\right)+\left(\frac{\mathrm{APT}}{\mathrm{AU}} \cdot 5+\frac{\mathrm{ANC}}{\mathrm{AU}} \cdot \text { EqNumV }\right)\right\}+0,30 \cdot(\text { EqNumDPI })+0,40 \cdot\left\{\left(\text { EqNum CA. } \frac{\mathrm{AC}}{\mathrm{AU}}\right)+\left(\frac{\mathrm{APT}}{\mathrm{AU}} \cdot 5+\frac{\mathrm{ANC}}{\mathrm{AU}} \cdot \mathrm{EqNumV}\right)\right\}+b_{0}^{1}
\end{aligned}
$$

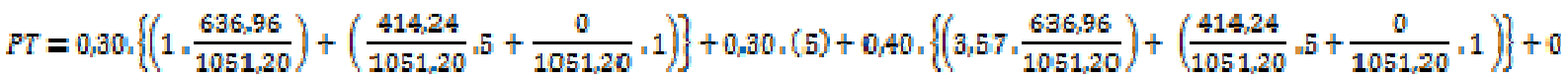

\section{PT $=4.03 \rightarrow$ clasificación de la edificación, nivel $B$.}

\section{ANÁLISIS CONFORME EL MÉTODO DE SIMULACIÓN}

\section{Modelo real}

El software elegido para la simulación fue el DesignBuilder, que contempla las exigencias impuestas por el reglamento. El modelo real de la edificación fue modelado conforme las directrices del RTQ - C, llevando en consideración todos los elementos arquitectónicos existentes, la orientación geográfica del edificio, los usos, desconsiderándose la sombra de los edificios vecinos por no haber ninguna edificación que produzca sombra relevante.

Resultado: el consumo total de energía de la edificación real a seguir indicado:

\begin{tabular}{|c|c|c|}
\hline Energypus oupar. & $\begin{array}{c}\text { Fuel Totals - Untitled, Building } 2 \\
\text { 1.3an-31 Dec:Amua } \\
\text { Yeas }\end{array}$ & Evauation \\
\hline Electricity $(\mathrm{kWh} / \mathrm{m} 2)$ & 64,55 & \\
\hline
\end{tabular}

Fig. 6 - Consumo total de la edificación - Modelo Real

\section{Modelos de referencia}

Los modelos de referencia deben considerar los ítems referenciados en la $R T Q-C$, tanto para el etiquetaje completo, como para el parcial. Posteriormente, con la obtención de los resultados de los modelos (real y los de referencia), éstos fueron comparados entre sí con la finalidad de clasificar la eficiencia del edificio real; las modificaciones hechas en los modelos de referencia se muestran a continuación: 


\begin{tabular}{|c|c|c|}
\hline Características del edificio & Modelo Real & Modelos de Referencia \\
\hline Geometría-dimensiones & Igual al edificio propuesto & Igual al edificio propuesto \\
\hline Orientación & Igual al edificio propuesto & Igual al edificio propuesto \\
\hline Carga interna & Igual al edificio propuesto & Igual al edificio propuesto \\
\hline $\begin{array}{l}\text { Padrón de usos (aparatos y } \\
\text { personas) }\end{array}$ & Igual al edificio propuesto & Igual al edificio propuesto \\
\hline $\begin{array}{c}\text { Sistema de condicionamiento del } \\
\text { aire }\end{array}$ & Igual al edificio propuesto & $\begin{array}{l}\text { Igual al edificio propuesto } \\
\text { Con COP mín. del nivel }\end{array}$ \\
\hline $\begin{array}{r}\text { Estructura externa/ envolvente } \\
\text { PAZ } \\
\text { PAFt } \\
\text { AVS y AHS } \\
\text { Factor solar } \\
\text { Transmisión térmica } \\
\text { Absorción solar }\end{array}$ & Igual al edificio propuesto & $\begin{array}{l}\text { Si existente } \rightarrow \text { PAZ }=2 \% \\
\text { Calcular por el IC } \\
\text { AVS }=\text { AHS }=0 \\
\text { Vidrio sencillo, } 3 \mathrm{~mm} \\
\text { FS }=0,87 \\
\text { Máx. } p / \text { la eficiencia deseada } \\
\text { Máx. } p / \text { la eficiencia deseada }\end{array}$ \\
\hline Sistema de iluminación & Igual al edificio propuesto & DPI máx. para la eficiencia deseada \\
\hline
\end{tabular}

La geometría de la edificación permanece igual; pero hay algunas alteraciones en los materiales de construcción, en el tamaño de las aberturas, se eliminan las de protecciones solares (cornisas y voladizos) y la sombra externa. Para la envolvente; estructura externa, las paredes y cubiertas deben seguir las siguientes composiciones:

Tabla 8 - Propiedades de las paredes y cubiertas de los modelos de referencia

\begin{tabular}{|c|c|c|c|c|}
\hline PROPRIEDADES & $\begin{array}{c}\text { ZONA } \\
\text { BIOCLIMATICA }\end{array}$ & NIVEL A & NIVEL B & NIVEL C Y D \\
\hline Ucob - Ambientes. Condicionados & 1 y 2 & 0.5 & 1.0 & \multirow{4}{*}{2.0} \\
\hline$\left[\mathrm{W} / \mathrm{m}^{2} . \mathrm{K}\right]$ & 3 a 8 & 1.0 & 1.5 & \\
\hline Ucob - Ambientes no Condicionados & 1 y 2 & 1,0 & 1,5 & \\
\hline$\left[\mathrm{W} / \mathrm{m}^{2} \cdot \mathrm{K}\right]$ & 3 a 8 & 2,0 & 2,0 & \\
\hline \multirow{3}{*}{ Upar $\left[\mathrm{W} / \mathrm{m}^{2} . \mathrm{K}\right]$} & 1 y 2 & 1.0 & 2.0 & 3.7 \\
\hline & 3 a 6 & \multicolumn{3}{|c|}{3.7} \\
\hline & 7 y 8 & \multicolumn{3}{|c|}{$3.7^{* *}$} \\
\hline \multirow{2}{*}{ Absorción Solar } & 1 & \multicolumn{3}{|c|}{ igual al modelo real } \\
\hline & 2 a 8 & \multicolumn{2}{|c|}{0.50} & 0.70 \\
\hline
\end{tabular}


* para paredes con capacidad térmica de hasta $80 \mathrm{KJ} / \mathrm{m}^{2} . \mathrm{K}$

** para paredes con capacidad térmica arriba de $80 \mathrm{KJ} / \mathrm{m}^{2} . \mathrm{K}$

Iluminación: Como el objeto de estudio ya posee clasificación $A$ para la iluminación, mas donde su potencia instalada es de $7.502 \mathrm{~W} / \mathrm{m}^{2}$, lo que es inferior al valor atingido por el $\mathbf{D P I}_{\mathbf{L}}$ Nivel A, fueron así verificados los prerrequisitos específicos a fin de mejorar la clasificación de la iluminación. Los modelos de referencia siguen las Potencias Instaladas conforme la tabla:

Tabla 9 - Densidad de potencia limite - Modelos de Referencia

\begin{tabular}{|c|c|c|c|c|}
\hline Función & $\begin{array}{c}\mathrm{DPI} \\
\text { Nivel A } \\
{\left[\mathrm{W} / \mathrm{m}^{2}\right.} \\
]\end{array}$ & $\begin{array}{c}\mathrm{DPI} \\
\text { Nivel B } \\
{\left[\mathrm{W} / \mathrm{m}^{2}\right]}\end{array}$ & $\begin{array}{c}\mathrm{DPI} \\
\text { Nivel C } \\
{\left[\mathrm{W} / \mathrm{m}^{2}\right]}\end{array}$ & $\begin{array}{c}\mathrm{DPI}_{\mathrm{L}} \\
\text { Nivel D } \\
{\left[\mathrm{W} / \mathrm{m}^{2}\right]}\end{array}$ \\
\hline $\begin{array}{c}\text { Despach } \\
\text { os }\end{array}$ & 9,7 & 11,2 & 12,6 & 14,1 \\
\hline Totales & $\begin{array}{c}9.699,6 \\
0\end{array}$ & $\begin{array}{c}11.199 \\
55\end{array}$ & $\begin{array}{c}12.599 \\
50\end{array}$ & $\begin{array}{c}14.099 \\
43\end{array}$ \\
\hline
\end{tabular}

Acondicionamiento de aire: los sistemas de aire acondicionado son del tipo Split; por el método de la simulación se debe mantener el mismo sistema,

pero cambiando el COP del sistema, para uno de mayor eficiencia, en ese sentido, se utiliza el COP referente a la clasificación siguiendo la próxima tabla.

Tabla 10 - Coeficiente de Performance (COP)

\begin{tabular}{|c|c|c|c|c|c|c|c|}
\hline \multirow{4}{*}{$\begin{array}{c}\text { Classes } \\
\mathrm{A}\end{array}$} & & $\pi$ & & & Data a & izaçäo & 11/2011 \\
\hline & \multirow{2}{*}{\multicolumn{3}{|c|}{$\begin{array}{l}\text { Coeficiente de eficiência } \\
\text { energética (W/W) }\end{array}$}} & \multicolumn{4}{|c|}{ Split Hi-Wall } \\
\hline & & & & \multicolumn{2}{|c|}{ Rotaçâo Fixa } & \multicolumn{2}{|c|}{ Rotação Variável } \\
\hline & 3,20 & $<C E E$ & & 226 & $25,3 \%$ & 122 & $83,0 \%$ \\
\hline B & 3,00 & <CEES & 3,20 & 178 & $19,9 \%$ & 17 & $11,6 \%$ \\
\hline C & 2,80 & <CEES & 3,00 & 294 & $32,9 \%$ & 8 & $5,4 \%$ \\
\hline D & 2,60 & <CEES & 2,80 & 159 & $17,8 \%$ & 0 & $0,0 \%$ \\
\hline$E$ & 2,39 & SCEES & 2,60 & 36 & $4,0 \%$ & 0 & $0.0 \%$ \\
\hline
\end{tabular}

\section{RESULTADOS DE LAS SIMULACIONES DE TODOS LOS MODELOS:}

\begin{tabular}{|c|c|c|c|c|c|}
\hline Consumo & Edificio Real & Nivel A & Nivel B & Nivel C & Nivel D \\
\hline Anual por $\mathrm{m}^{2}\left[\mathrm{KWh} / \mathrm{m}^{2}\right]$ & 64,55 & 65,87 & 67,07 & 79,85 & 82,02 \\
\hline Total anual $[\mathrm{MW} / \mathrm{h}]$ & 248,18 & 263,83 & 269,46 & 306,98 & 315,35 \\
\hline
\end{tabular}

La puntuación total (PT) de los edificios simulados es calculada por la ecuación:

$$
\mathrm{PT}=\mathrm{EqNumS}+b_{0}^{1}
$$

$$
\mathrm{PT}=5+0=5 \rightarrow \text { Clasificación } A
$$

\section{CONCLUSIONES}

Desde el punto de vista de la aplicación profesional de la reglamentación, y por esta ser su primera edición, se ha verificado en esta tesina la existencia de algunas partes en que hay puntos débiles en las metodologías y los aportes que sin embargo ellas sustentan. $Y$ aunque el referente trabajo ilumine $y$ denote una pequeña muestra de su potencialidad ya que no sería verosímil analizar toda la gama de parámetros disponibles por sus niveles de complejidad y dada la gran variedad diversidad de variables de las edificaciones, el saldo es muy positivo. Centrándose en el estudio de caso aplicado de un edificio comercial, en altura, ubicado en el sur de Brasil, se puede citar que los resultados de este ensayo muestran:

- que la reglamentación en foco es fiel a los conceptos mundiales de eficiencia energética y sus propuestas metodológicas, para su aplicación, denotan una relevancia 
directa con los conceptos de la arquitectura bioclimática.

- que por las metodologías aplicadas, las observaciones han sido de gran variedad y han tendido a analizar, positivamente, la factibilidad del manejo y utilización de las herramientas disponibles.

Del Método Prescriptivo es menester destacar algunos aspectos que por ahora no han sido ponderados, tal como la desconsideración del norte geográfico en los cálculos de la estructura externa de las edificaciones (envolvente), así como de elementos del entorno. Asimismo, la determinación de los ángulos de sombra horizontales no tiene correlación con la trayectoria solar del lugar; éstos son ponderados y provenientes de los dos vértices y el análisis solar del sitio de la construcción - en contexto con la edificación - podría ser más detallado. Después de la aplicación exhaustiva de ecuaciones, cálculos y levantamiento de datos, se puede inferir que la herramienta 'prescriptiva' se torna relativamente difícil de ser usada, exigiendo un largo periodo de tiempo para organizar las normas, datos e informaciones que deben ser necesariamente verificadas. Tiende, eso sí, a ser el método más utilizado en virtud de dar un mejor acceso profesional a quienes se relacionan con el área en foco, dado que la Simulación (como método, descrito "ut infra") exige conocimiento y uso de un software especializado, lo que no es - hoy por hoy una realidad tangible en Brasil. Esto, en virtud de que la mayoría de los Estudios de Arquitectura e Ingeniería no acceden a esta herramienta dados los altos costos de su implementación y por la necesaria dedicación - en horas de estudio - para su empleo formal. Por ahora, su aplicación se limita fundamentalmente a laboratorios universitarios y a grandes Estudios de construcción civil.

El Método de la Simulación es más oneroso. Se inclina a ser más fiable, pero por su vez, también deja a desear en algunos ítems, como el desconsiderar el espesor de las escuadrías en los modelos, la infiltración de aire en las aberturas y otros aspectos referentes a éstas. El cálculo del nuevo PAFt es un tanto cuanto complicado, pero evidencia la utilización de la insolación en el modelo. Para el acondicionamiento de aire el método no analiza la capacidad total del sistema, una vez que incorpora apenas el valor del COP como determinante de los modelos de referencia. La Simulación es una herramienta potente (un software que emplea mucha de la capacidad de un ordenador) y así, se debe tener en cuenta que, cuanto más uso y manejo de informaciones exista, o cuanto mayor sea la complexidad de la edificación a ser apreciada, todo ello ha de contribuir a disminuir la velocidad del proceso y ha de requerir, por tanto, ordenadores potentes para realizarla.

Por último, se ha verificado que las clasificaciones entre los métodos no se equivalen. El Método Prescriptivo clasifica el estudio de caso como de nivel $B$ de eficiencia energética, en tanto y en cuanto la Simulación la determina en un nivel $A$. Esa diferencia entre los resultados se ha dado porque el primero procura regular requisitos mínimos de prerrequisitos específicos. En puntos importantes, como la transmisión térmica de la cubierta de la torre comercial, ello ha sido un factor determinante para que la clasificación de la envolvente de la estructura externa bajase para $E$. $Y$ hay un área irrelevante - en relación a toda la envolvente - que, por el cálculo del Índice del Envoltorio (ICenv), quedó con clasificación $A$, verificándose así que por la Simulación, y por ser ésta una herramienta más precisa, tiende a ser más fiel a la situación real. Se puede concluir entonces, que el prerrequisito de la transmisión térmica de la cubierta podría ser repensado en función de edificaciones donde éstas ocupen una pequeña área en relación al conjunto de la edificación, con relación directa a los Factores de Forma y de Altura.

Finalmente, con todas las informaciones evaluadas, ha quedado patente la intención de que futuramente haya un padrón en la clasificación de las características de los materiales empleados en la construcción civil, con la finalidad de ayudar a los profesionales en la selección de la metodología y en los cálculos de eficiencia energética. Ha quedado también evidente la necesidad de adicionar prerrequisitos de construcción más rigurosos en las próximas ediciones de la reglamentación, que tengan a la arquitectura pasiva como modelo, y también medidas que minimicen la utilización de la energía aún en el cantero de obras, y/o que puedan ser analizadas ya en la etapa proyectual. 